\title{
Phylogenomic analysis of viral genomes assembled from Juan de Fuca Ridge flank basalt-hosted crustal fluids
}

\author{
C. SPOTKAEFF ${ }^{1 *}$, M. S. RAPPE ${ }^{2}$, S.P. JUNGBLUTH ${ }^{2}$, G. \\ STEWARD $^{3}$, O. D. NIGRO ${ }^{1}$ \\ ${ }^{1}$ Hawaii Pacific Univ., Honolulu, HI 96813, USA \\ (*correspondence: cspotkae@my.hpu.edu) \\ ${ }^{2}$ Hawaii Institue of Marine Biology, School of Ocean and \\ Earth Science and Technology, Univ. of Hawaii at \\ Manoa, Kaneohe, HI 96744, USA \\ ${ }^{3}$ Department of Oceanography, Daniel K. Inouye Center for \\ Microbial Oceanography, Univ. of Hawaii at Manoa, \\ Honolulu, HI 96822, USA
}

Over the last decade, research in the oceanic deep subsurface has been advanced through the study of basaltic basement fluids retrieved from boreholes that have been outfitted with Circulation Obviation Retrofit Kit (CORK) observatories. Significant effort has been given to understanding the microbial community inhabiting the crustal fluid, as its contribution to biogeochemical cycling in the basaltic crust is not well understood [1]. Although there are ongoing studies that detail the microbial community dynamics at Juan de Fuca Ridge (JdFR), limited studies have been focused on the viral community [2]. In these nutrient-limited, geothermallyheated environments where eukaryotic life is most likely to be absent, evolutionary pressure from viruses may be playing a crucial role in shaping microbial populations [3]. In this study, we used a customized in situ ultrafiltration system combined with a physical fractionation of intact viral particles to create density-fractionated metagenomes from boreholebasement fluids at JdFR. From these metagenomes, we have identified and annotated 25 complete, circularized viral genomes, ranging in size from $2,656 \mathrm{bp}$ to $77,875 \mathrm{bp}$. In addition to viral hallmark genes, some of these genomes feature auxiliary metabolic genes (AMGs) associated with pathways such as assimilatory sulfate reduction, nucleotide metabolism and cysteine and methionine metabolism. Anaylsis of CRISPR spacer sequences from previously described JdFR microbial metagenomes [1], the hosts for six of these viruses have been identified. These hosts include members of novel clades of Archaeoglobus, Bathyarchaeaota, Euryarchaeota and Aminicenantes. These viral genomes will further shed light on the role of the virosphere in driving host diversity which is not well understood.

[1] Jungbluth et al. (2016) ISME J. 10, 2033-2047. [2] Nigro et al. (2017) MBio. 8, 1-15. [3] Anderson et al. (2013) Rev. mineral geochem 75, 649-675. 